\title{
Muon tomography for railway tunnel imaging
}

\author{
L. F. Thompson $\oplus^{*}$, J. P. Stowell, and S. J. Fargher \\ Department of Physics and Astronomy, University of Sheffield, Sheffield, S3 7RH, United Kingdom \\ C. A. Steer, K. L. Loughney, and E. M. O'Sullivan \\ Applied Physics, St Mary's University, Waldegrave Road, Twickenham TW1 4SX, United Kingdom \\ J. G. Gluyas \\ Department of Earth Sciences, Durham University, South Road, Durham, DH1 3LE, United Kingdom \\ S. W. Blaney and R. J. Pidcock \\ Central Alliance, Alliance House, South Park Way, Wakefield, WF2 OXJ, United Kingdom
}

(Received 14 June 2019; revised manuscript received 8 November 2019; accepted 5 March 2020; published 8 April 2020)

\begin{abstract}
Cosmic ray muon radiography utilizes highly penetrating cosmic ray muons to image the density profile of an object of interest. Here we report on a trial to use a portable field-deployable cosmic ray muon tracking system in order to image the whole overburden of a UK railway tunnel with short-duration scans (c. 30 min). An unknown overburden void was identified and, after trial, confirmed by railway authorities. These experiments demonstrate the identification of hidden construction shafts with high levels of statistical significance as density anomalies within the data.
\end{abstract}

DOI: 10.1103/PhysRevResearch.2.023017

\section{RAILWAY INFRASTRUCTURE AND MAINTENANCE}

The United Kingdom's railway network is currently estimated to extend to more than 20000 miles of track and more than 30000 tunnels, viaducts, and bridges. Much of the current infrastructure was constructed in the 19th century and is still in use today [1], which is made possible by a robust safety regime.

Railway tunnels present significant challenges in assuring their safety over long time periods. Hidden degradation of tunnel linings, where water ingress, differential settlement, and other phenomena can lead to so-called voids opening up.

Similarly, large historic construction shafts, used by the 19th century engineering teams to speed up completion, are also of concern. When a railway tunnel was finished, some vertical construction shafts would be used for ventilation and some sealed over within the tunnel and surface and left. The 1953 hidden shaft collapse in Swinton, near Manchester, England, starkly illustrates that a lack of construction shaft identification and then remediation can cause fatalities for urban populations at the surface [2].

\footnotetext{
*1.thompson@ sheffield.ac.uk

Published by the American Physical Society under the terms of the Creative Commons Attribution 4.0 International license. Further distribution of this work must maintain attribution to the author(s) and the published article's title, journal citation, and DOI.
}

Searching for and monitoring tunnel voids can be timeconsuming and may involve many personnel working in potentially hazardous environments. Investigations typically require intrusive drilling into the tunnel lining to discern the presence, or not, of hidden voids and poorly back-filled shafts. Intrusive drilling investigations may cause instabilities and partial failure for marginal linings or, as a shaft is drilled into, the outpouring of collected groundwater. The possibility of an alternate noninvasive technique for rapidly and accurately detecting overburden changes without placing personnel at risk is clearly advantageous.

Cosmic ray muon radiography poses an ideal solution to this problem, provided imaging systems are capable of meeting the strict timing and portability requirements required for working in live railway tunnels. Cosmic ray muons are also highly penetrating, with significant fluxes capable of passing through hundreds of meters of rock and soil overburden. As a result, the technique has previously been successfully applied to the imaging of Egyptian pyramids [3,4], nuclear reactors [5], volcanoes [6], and an underground tunnel [7].

The technique is particularly suited to measuring overburden in railway tunnels, in which detectors can always be placed directly below the topography to be imaged. It is expected that the technique discussed here is applicable to a wide range of tunnel sizes and depths, provided the total exposure time for each run can be modified to ensure enough data are collected at each sample point. The desired measurement significance, tunnel depth, and other instrumental factors determine the shaft "signal" to no-shaft "background" rates, and ultimately the count time required. The limitations to the 


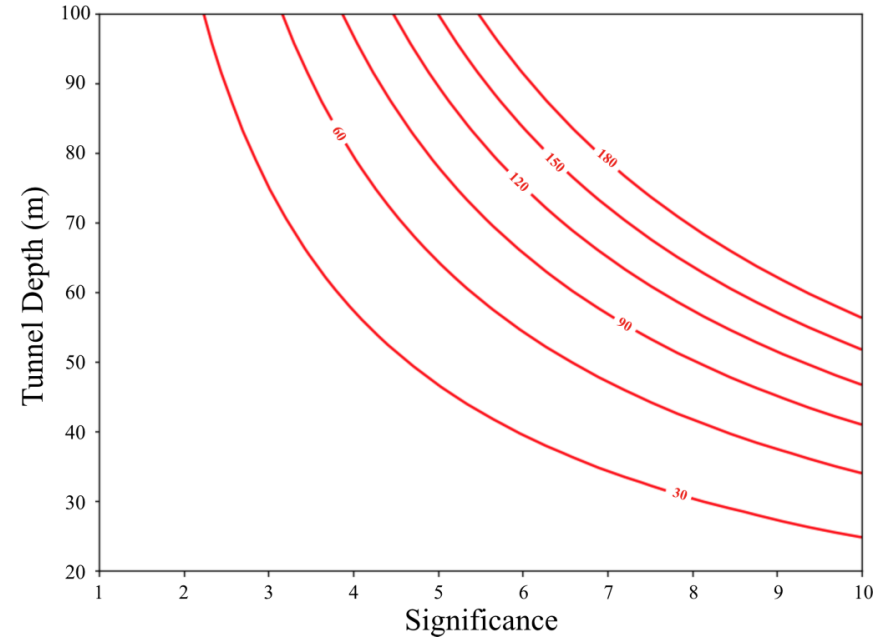

FIG. 1. Significance of an open shaft observation as a function of tunnel depth (overburden) and measurement time.

technique are therefore set by practicality (and requirements) of the application to provide a useful measurement within a set period of time.

Comparisons of the simulated attenuation of muons assumed to be traveling through a uniform density can be used to infer the sensitivity of the technique. Figure 1 presents these results as a function of time versus statistical significance, defined as $(S-B) / \sqrt{B}$, where $S$ corresponds to signal (rate with shaft present) and $B$ to background (rate without the shaft). Curves on the figure denote fixed measurement time (in minutes) and this enables a measurement time to be determined for a fixed overburden and required significance. These simulations can also be used to infer an approximate depth of overburden along a line of sight based on the experimentally measured data.

\section{THE ALFRETON OLD TUNNEL}

Situated between Alfreton and Langley Mill in Nottinghamshire, England, Alfreton Old Tunnel is a disused straight railway tunnel which is $770 \mathrm{~m}$ long along its axis from the

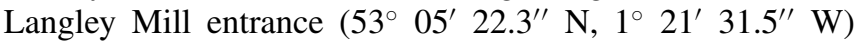

to the Alfreton entrance $\left(53^{\circ} 05^{\prime} 43.5^{\prime \prime} \mathrm{N}, 1^{\circ} 21^{\prime} 54.6^{\prime \prime} \mathrm{W}\right)$. The Old Tunnel was constructed in 1862 and was built using three known construction shafts, which were retained for ventilation; these open shafts are visible both from the surface and from inside the tunnel. Alfreton Old Tunnel is sited $28 \mathrm{~m}$ west of the New Tunnel, which was constructed in 1902 and is still in use. Unlike the Alfreton New Tunnel, where temporary construction (and subsequently hidden) shafts are well documented [8], no records of temporary construction shafts can be found for the Old Tunnel.

The area above the tunnel is undeveloped, consisting mainly of scrub growth other than the main A38 dual carriageway and a minor road, which both cross over the line of the tunnel. Between the Alfreton entrance and the A38, spoil mounds (from the tunnel's construction) are apparent, up to approximately $7 \mathrm{~m}$ high. Figure 2 depicts the results of a topographical survey of the tunnels and surrounding area. A subset of the data from this survey, specifically those data taken along a straight line directly above the tunnel at its maximum height (the so-called "crown line"), were used to estimate the thickness of rock directly above the detector at each measurement point. Using a uniform rock density based on geological survey information for the site, muon attenuation simulations were used to artificially reduce the measured open-sky rate. This calculation, referred to as the expected rate in subsequent plots, represents the total flux of muons one would expect to see in the detector at each measurement point if no significant density anomalies are present in the overburden.

\section{INSTRUMENTATION, DATA ACQUISITION, AND TRIGGERING}

The muon tomography system design focused on a number of features key to field deployment including robustness, ease of operation, and low power budget. The system comprises two horizontal layers of EJ-200 plastic scintillator. The upper layer is segmented into six independent rectangular bar detectors, each $90 \times 15 \times 4 \mathrm{~cm}$ in size. Similarly, the lower layer is segmented into three independent square paddle detectors, each $30 \times 30 \times 4 \mathrm{~cm}$ in size as depicted in Fig. 3. Each individual detector is contained in a light-tight housing and

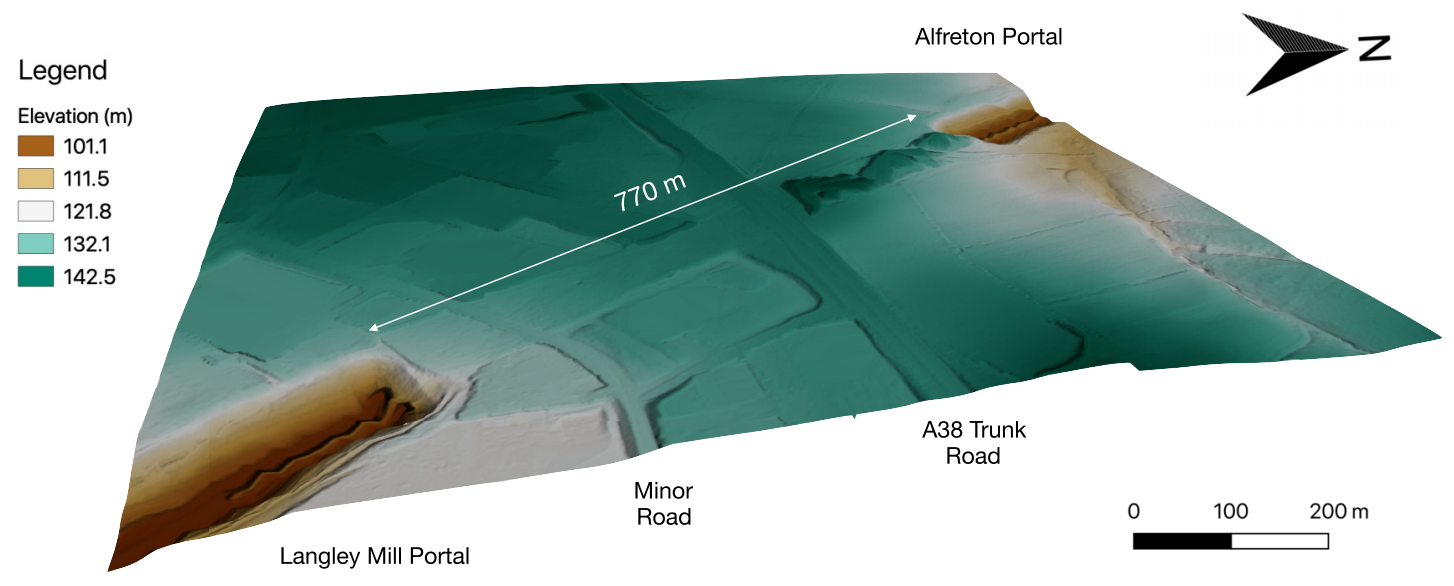

FIG. 2. Representation of the Alfreton Old and New Tunnels and surrounding using open-source LiDAR data [9]. 


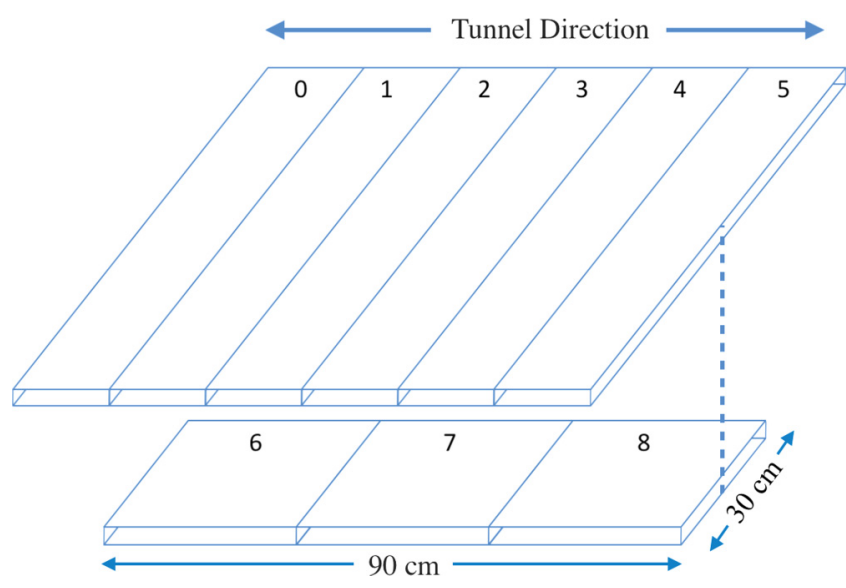

FIG. 3. Schematic of the scintillator configuration; the dotted line represents the vertical in the system.

coupled to a photomultiplier tube for signal readout. In both layers, detectors are placed next to one another with their longest side running perpendicular to the tunnel length and their shortest dimension running vertically. The top layer is fixed $76 \mathrm{~cm}$ above the bottom, giving the system a $100^{\circ}$ field of view along the axis of the tunnel, and a $76^{\circ}$ field of view along its width.

Data from each individual detector is processed using a CAEN DT5740 digitizer, sampling each channel at $62.5 \mathrm{MHz}$ [10]. The digitizer is triggered from a built-in hardware-based logical OR signal of the lower three detector paddles. The effective detection area of the system is therefore dictated by the area of the combined lower detectors $(30 \times 90 \mathrm{~cm})$. Once a trigger is registered, the signal from each of the nine detectors is read out and a second software trigger is formed from a logical AND of any upper layer bar with any lower layer paddle within a time window of $64 \mathrm{~ns}$. Specifically, for a detector to be considered in the software trigger, it has to observe a signal above a predefined threshold. These thresholds are optimized for each detector individually to maximize muon triggering efficiency, while rejecting background signals such as dark noise, etc. After threshold optimization, the estimated efficiency for detecting through-going muons within a single detector was between $88 \%$ and $98 \%$. For each trigger, an event record is saved containing an event time and pulse height for every triggered channel. In effect, this permits 18 different solid angle regions to be sampled simultaneously.

As the system needed to be operated within the tunnel, it was required to be portable and to be capable of being run remotely with no main voltage. With a total footprint of $1.3 \times 1.3 \mathrm{~m}$, the system is small enough to be installed and operated in the back of a commercially available Ford Transit Custom 270 van. Overall, the system's power requirements are typically $50 \mathrm{~W}$. Consequently, a large-capacity battery and $\mathrm{DC} / \mathrm{AC}$ converter provide sufficient power to run the full system for $50 \mathrm{~h}$ without recharging the battery.

\section{ALFRETON FIELD TRIAL}

The muon flux rate drops significantly as a function of overburden, and therefore initial feasibility studies were performed to understand the minimum exposure time required to identify voiding or significant density changes with a high reliability. Simulations of muons generated with the CRY library [11], propagated using GEANT4 [12] through a threedimensional (3D) reconstruction of the Alfreton Old Tunnel overburden, found that exposure times as short as $30 \mathrm{~min}$ are sufficient to identify open voids and voiding behind the tunnel lining. These simulations were used to inform the required exposure times necessary for scanning the entire length of the Alfreton Old Tunnel.

The Alfreton Old Tunnel data-collection campaign consisted of up to 8-h shifts for 12 days. Each day, the system was transported to the site and assembled in the morning, mimicking operating conditions on a live railway tunnel. Before operation in the tunnel, or in some cases overnight, several open-sky measurements were taken of the unobstructed muon flux through the system for later calibration. During data collection, the system was positioned relative to existing tunnel distance markers that are fixed to the walls at nominal 20 - $\mathrm{m}$ intervals. A laser range finder was then used to further constrain the system location to within $20 \mathrm{~cm}$ of a chosen position along the tunnel length. Similarly, the laser range finder was used to position the system midway along the tunnel cross section to ensure the apex of the tunnel was kept in the center of the system's field of view. Data were taken along the entire tunnel at $10-\mathrm{m}$ intervals, with more than 150 runs being taken in total. For scans around overburden regions of interest, the distance intervals were reduced to $5 \mathrm{~m}$. At each position, 20 or $30 \mathrm{~min}$ of muon flux data were taken, as well as pressure and temperature readings in 30-s increments.

\section{DATA ANALYSIS AND INTERPRETATION}

The principal analytical tool comprises summing software triggers across the full system as described in Sec. III and converting this to a muon flux rate per $30 \mathrm{~min}$. Figure 4 depicts the variation of this muon flux rate as a function of distance along the tunnel relative to the Langley Mill portal. The errors displayed on the rate for each data run are purely statistical. The broad features of this figure concur with the expected rate derived from the topographical survey discussed in Sec. II. A rapidly reducing muon flux at both tunnel entrances was observed, as the overburden increases, as well as a minimum in the muon flux at $400 \mathrm{~m}$ from the Langley Mill entrance, where the overburden is the greatest.

Superimposed on Fig. 4 is the known location of the three open shafts (in light gray). The variation in the rate at the open shafts is very clear and highly statistically significant (up to 10 standard deviations when compared with the average trend of the data either side). Any hidden shaft would be expected to create similar variations in the data. Figure 5 provides an enlargement of the data around the open shaft at $222.5 \mathrm{~m}$ as an indication of the variation of the flux under the open shafts. Since the shaft is comparable in size to the data-collection intervals, a sharp increase is seen when directly under the shaft relative to the points directly either side.

Returning to Fig. 4, while there is general agreement, it is clear that there are several areas where there is disagreement between the expected and measured rates. Note that the discontinuity in the data at $350 \mathrm{~m}$ is known to be due to a 


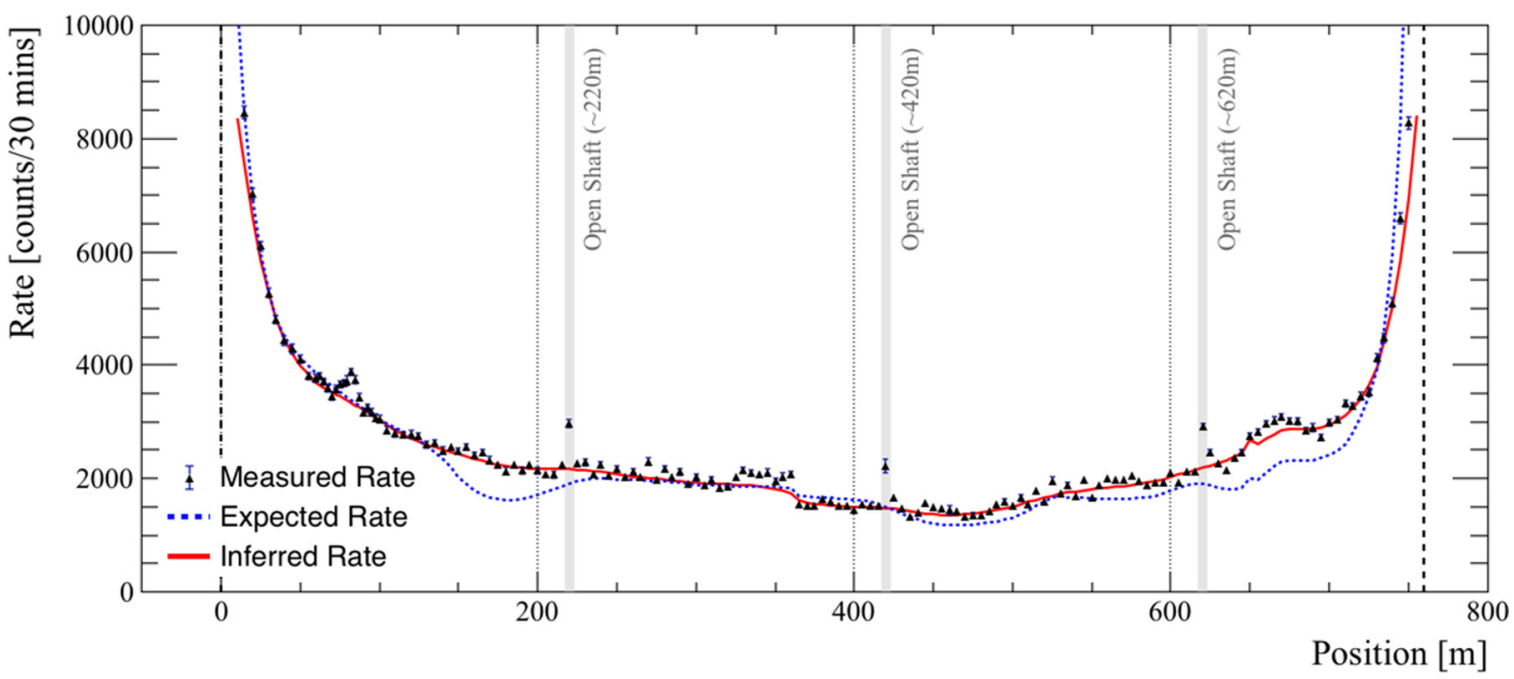

FIG. 4. Comparison of measured muon flux rate with the expected rate (from topographical information) and inferred rate along the full length of the tunnel. Distances measured from the Langley Mill entrance.

change in efficiency as a result of exchanging a faulty cable and is not related to an overburden change. Between 150 and $200 \mathrm{~m}$ and also between 600 and $700 \mathrm{~m}$, the measured muon flux rate is higher than expected from the overburden measurement, indicating a region of lower than expected density or overburden in the ground above the tunnel.

After a careful comparison of all possible bar and paddle trigger combinations in this region, it was concluded that the observed discrepancy was due to some larger scale effect and not the presence of a localized high-density feature. This conclusion is supported by figures such as those in Fig. 6 where it can be observed, e.g., that any excess around 650-700 m appears as a simple scaling of the open sky rate, suggesting that there are no small localized density features. Contrast this with the observations at $620 \mathrm{~m}$, where clear excess is seen on multiple detector paddles that are pointing directly up. Since the topographic data were sampled in a straight line along

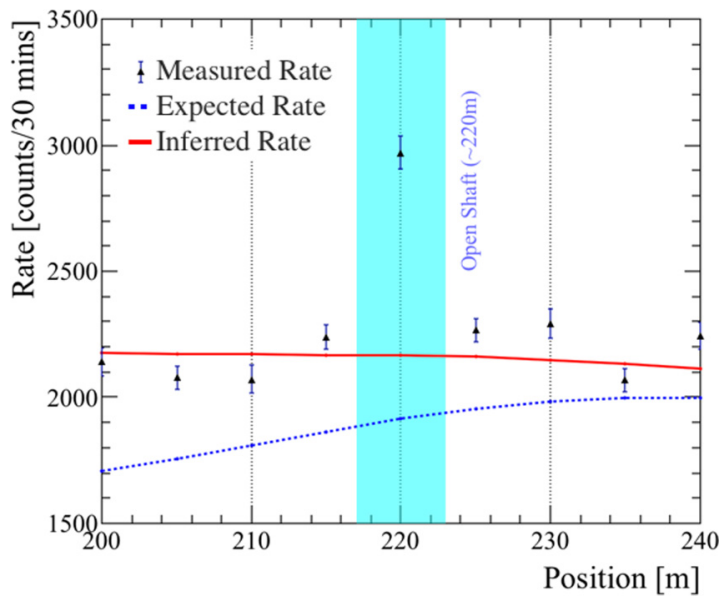

FIG. 5. Comparison of measured muon flux rate with the expected rate and inferred rate around the known open shaft at $220 \mathrm{~m}$ from the Langley Mill portal end of the tunnel. The inferred rate is a refined estimate using the data to update the overburden estimate as discussed below. the top of the tunnel, it is expected that if additional density variations due to the landscape or overburden geology exist away from this line but are still within the field of view of the detector, such features would become apparent in the data. This could be corrected for by performing a higher precision topography scan $(\approx 10 \mathrm{~m}$ resolution) of the overburden above the tunnel, covering the entire angular field of view of the detector. However, in some cases, where access is limited or historic knowledge has been lost, such fine-scale topography information may be unavailable. Therefore, it was advantageous to consider if muon tomography could be used to identify voiding with only limited access to this information.

The level of redundancy in the system data, which simultaneously records 18 angular bins, provides the opportunity to address the question of the erroneous expected muon flux from the topographical survey. By making an assumption on the average surrounding rock density, the decrease in muon flux rates compared with the open sky measurement for a given bar and paddle trigger combination can be used to infer the rock thickness along a specific line of sight. Overlaying information from multiple lines of sight and positions can be used to triangulate the average surface height at a given position. The mean surface height for any given position along the tunnel provides an estimate of the overburden given an assumed rock density. In cases where the rock density is not well known, or expected to vary significantly, these inferred overburden estimates can be combined with a limited number of manually measured overburden points to further refine the overburden estimate determined in this way. Furthermore, using a wide binning (e.g., $\approx 20 \mathrm{~m}$ ) allows the overburden to be estimated with weak dependence on small scale features of interest such as $\approx 5 \mathrm{~m}$ shafts. When the overburden is calculated in this way, it is possible to infer an updated rate estimate as seen in Fig. 4 (labelled "inferred rate"), which removes the large-scale features observed in the previous estimate. This technique is a promising one for general overburden calculations in areas with limited ground-level access.

In addition to the three known open shafts, there are additional points along the tunnel where there is poor agreement 

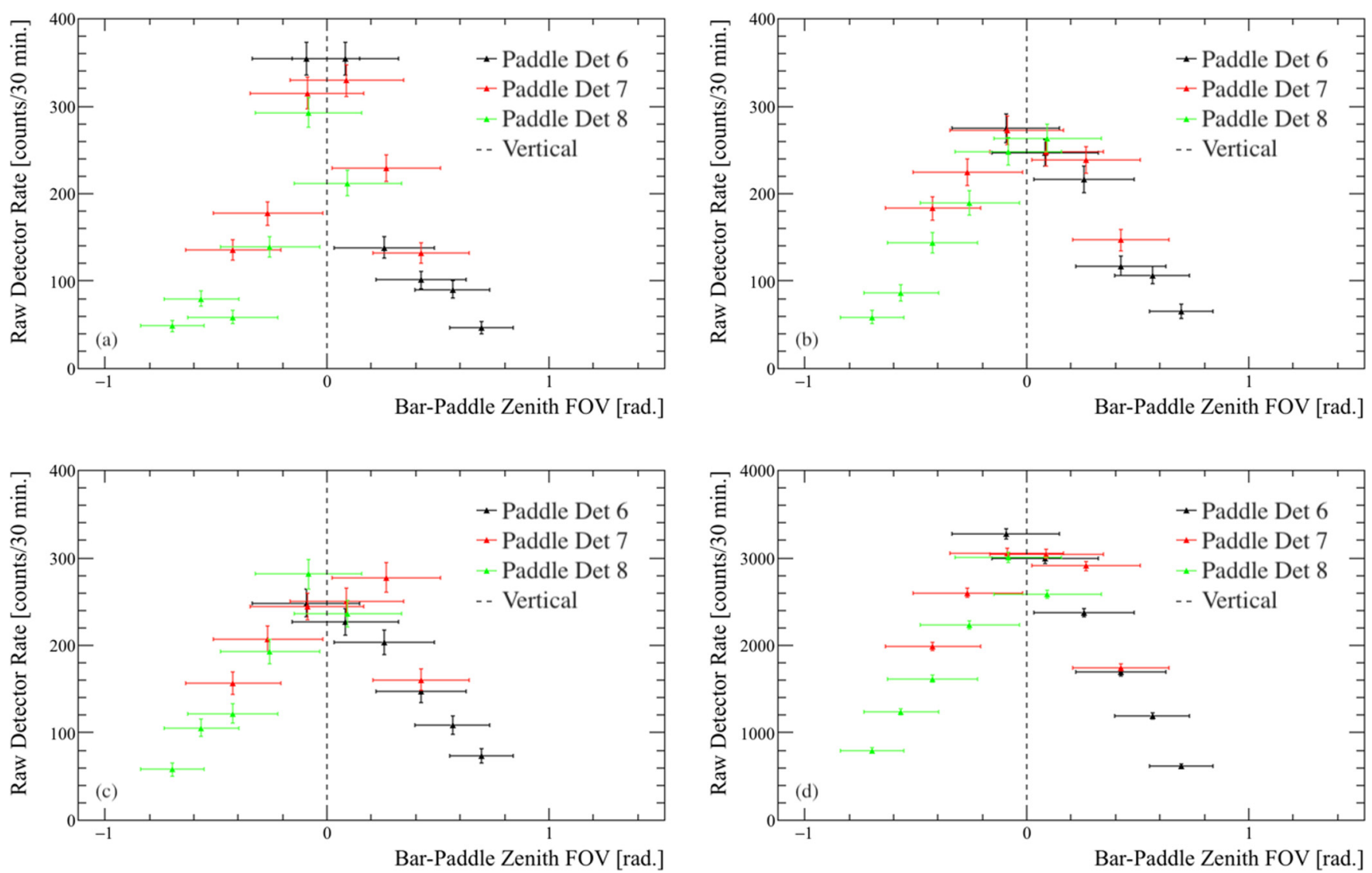

FIG. 6. Muon flux rates on upward-looking bar-paddle combinations for the following points along the tunnel: $625 \mathrm{~m} \mathrm{(a),} 650 \mathrm{~m}$ (b), $700 \mathrm{~m}$ (c), and open sky (d) as a function of the field of view of those detector combinations.

between observed and inferred muon flux rates which may be indicative of hidden features that result in overburden changes. One in particular, that at $80 \mathrm{~m}$, was investigated by introducing additional scan points at finer $2.5-\mathrm{m}$ spacings. As illustrated in Fig. 7, at $80 \mathrm{~m}$ there is a statistically significant excess in the muon flux compared with both the expected and

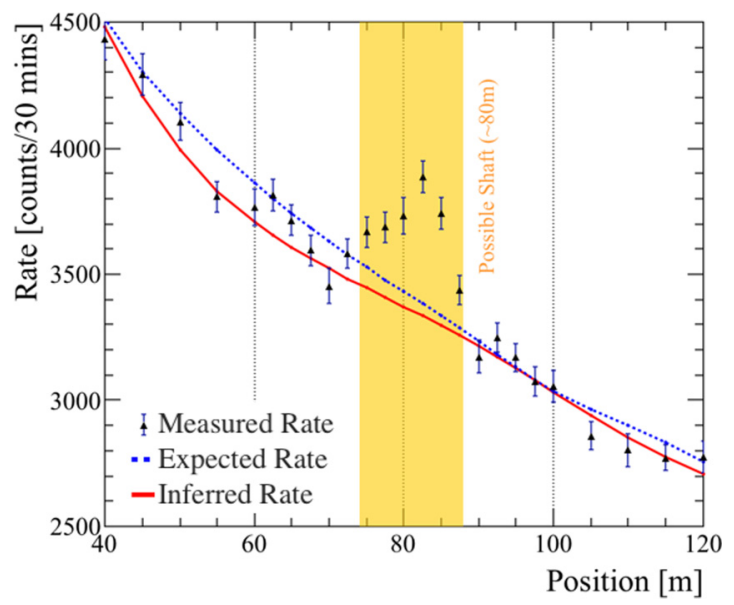

FIG. 7. Comparison of measured muon flux rate with the expected rate and inferred rate around the suspected hidden shaft at $80 \mathrm{~m}$. inferred flux; however, unlike the case for the open shafts, this feature appears to be broader in extension, which may imply a hidden shaft that has undergone some degradation, such as material infall from the sides and/or partial infill. Following disclosure of the results of this blind test to the rail authorities, the authors have subsequently been made aware of pre-existing concerns that there is a hidden void in this area. These suspected voided regions are also at similar positions, with respect to the tunnel entrances, as the known hidden shafts in the Alfreton New Tunnel, providing further confidence. Of note is that both tunnels therefore appear to have concealed shafts within $80 \mathrm{~m}$ of both portal entrances, rather than toward the middle of the tunnel. It should be stressed that this information was not made available to the authors at the time of the trial.

\section{CONCLUSION}

The first whole-length overburden measurements of a railway tunnel have been performed to search for potentially dangerous voids, such as hidden construction shafts. Cosmic ray muon radiography has been demonstrated as a viable technique for the identification of voiding and significant density changes inside railway tunnel overburden. A portable system with limited angular resolution has been shown to be capable of detecting open shafts with high statistical significance inside the Alfreton Old Tunnel in the UK within a 
short exposure time of only $100 \mathrm{~h}$. In this blind test, one hidden void above the Alfreton Old Tunnel was identified and, after trial, confirmed by rail authorities. The use of redundant data to predict the tunnel's overburden illustrates the power of the cosmic ray muon radiography technique as a practical method for overburden mapping even when full topographical information is not available.

\section{ACKNOWLEDGMENTS}

The authors acknowledge the assistance of Ian Wilson from Network Rail and Dave Thomas from AMCO in the facilitation of this field trial as well as support from awards from both EPSRC and STFC Impact Acceleration Accounts.
[1] H. J. Pragnell, Early British railway tunnels: The implications for planners, landowners, and passengers between 1830 and 1870, Ph.D. thesis, University of York, York, UK, 2016; http: //etheses.whiterose.ac.uk/16826/.

[2] https://en.wikipedia.org/wiki/Clifton_Hall_Tunnel.

[3] L. W. Alvarez et al., Search for hidden chambers in the pyramids, Science 167, 832 (1970).

[4] K. Morishima et al., Discovery of a big void in Khufu's pyramid by observation of cosmic-ray muons, Nature (London) 552, 386 (2017).

[5] H. Miyadera et al., Imaging Fukushima Daiichi reactors with muons, AIP Adv. 3, 052133 (2013).

[6] H. K. M. Tanaka, T. Kuságaya, and H. Shinohara, Radiographic visualization of magma dynamics in an erupting volcano, Nat. Commun. 5, 3381 (2014).
[7] E. Guardincerri et al., 3D cosmic ray muon tomography from an underground tunnel, Pure Appl. Geophys. 174, 2133 (2017).

[8] E. F. C. Trench, Alfreton second tunnel, Min. Proc. Inst. Civil Engin. 161, 116 (1905).

[9] Contains public sector information licensed under the Open Government Licence v3.0.

[10] https://www.caen.it/products/dt5740/.

[11] C. Hagmann et al., Cosmic-ray shower generator (CRY) for Monte Carlo transport codes, in Proceedings of IEEE Nuclear Science Symposium Conference Record, 2007. NSS'07, Vol. 2, Honolulu, Hawaii, USA, (2007), pp. 1143-1146.

[12] S. Agostinelli et al., Geant4-a simulation toolkit, Nucl. Inst. Meth. A 506, 250 (2003). 